\section{Low-resolution Gamma-ray Spectrometry for an Information Barrier Based on a Multi-Criteria Template-Matching Approach}

\author{
Malte Göttsche, Janet Schirm, Alexander Glaser \\ Princeton University
}

Preprint submitted to Elsevier
Abstract

Gamma-ray spectrometry has been successfully employed to identify unique items containing special nuclear materials. Template information barriers have been developed in the past to confirm items as warheads by comparing their gamma signature to the signature of true warheads. Their development has, however, not been fully transparent, and they may not be sensitive to some relevant evasion scenarios. We develop a fully open template information barrier concept, based on low-resolution measurements, which, by design, reduces the extent of revealed sensitive information. The concept is based on three signatures of an item to be compared to a recorded template. The similarity of the spectrum is assessed by a modification of the Kolmogorov-Smirnov test to confirm the isotopic composition. The total gamma count rate must agree with the template as a measure of the projected surface of the object. In order to detect the diversion of fissile material from the interior of an item, a polyethylene mask is placed in front of the detector. Neutrons from spontaneous and induced fission events in the item produce $2.223 \mathrm{MeV}$ gamma rays from neutron capture by hydrogen-1 in the mask. This peak is detected and its intensity scales with

October 4, 2016 
the item's fissile mass. The analysis based on MCNP Monte Carlo simulations of various plutonium configurations suggests that this concept can distinguish a valid item from a variety of invalid ones. The concept intentionally avoids any assumptions about specific spectral features, such as looking for specific gamma peaks of specific isotopes, thereby facilitating a fully unclassified discussion. By making all aspects public and allowing interested participants to contribute to the development and benchmarking, we enable a more open and inclusive discourse on this matter.

Keywords: gamma-ray spectrometry, information barrier, template approach, nuclear warhead verification, nuclear disarmament, nuclear arms control, prompt-gamma activation analysis

\section{Background}

Current nuclear arms-control agreements between the United States and Russia limit the number of deployed strategic nuclear weapons each party can have. Future nuclear arms-control agreements, however, are likely to also place limits on the number of nuclear weapons and warheads that a country can maintain in its arsenal. For example, the 2010 U.S. Nuclear Posture Review Report recommended that the United States should engage Russia in negotiations aimed at "achieving substantial further nuclear force reductions and transparency that would cover all nuclear weapons - deployed and non-deployed, strategic and nonstrategic" [1]. Such agreements would require the inspection and verification of warheads that are in storage or queued for dismantlement. To accomplish this task, viable verification approaches must be available to confirm the authenticity 
of nuclear components while also protecting classified information $[2,3,4]$. One such approach would be to analyze the gamma spectrum originating from an item identified for verification, and - through the use of an information barrierconfirm or deny the identity of the item as a valid nuclear component without otherwise revealing any information about the item.

Several information barriers have been developed based on the so-called attribute method, whereby a given set of attributes, such as mass thresholds and isotopic ratios, are used to characterize (classified) nuclear components. These systems typically require high-resolution detectors, such as high-purity germanium detectors, to perform this task. Prominent attribute systems are the "Trusted Radiation Attribute Demonstration System" (TRADS) [5], the "Attribute Verification System with Information Barrier for Plutonium with Classified Characteristics utilizing Neutron Multiplicity Counting and HighResolution Gamma-ray Spectrometry" (AVNG) [6] developed as part of the Trilateral Initiative between the United States, Russia, and the International Atomic Energy Agency and, more recently, the information barrier developed as part of the UK/Norway Initiative (UKNI) [7]. A second approach, called template-matching, has been developed in parallel. The template approach does not seek to determine absolute characteristics of the inspected item, such as plutonium mass or isotopics; instead, this complementary method seeks to detect significant differences between two items, for example by means of gamma spectroscopy, without seeking to determine the origin for these differences. Few such systems have been proposed and built; among these, the Trusted Radi- 
ation Inspection System (TRIS) developed at Sandia National Laboratories is the most prominent and widely tested one [8]. While the general strategy of TRIS has been described in the literature, few relevant details of the developed algorithm have been published. For a more detailed overview of both attribute and template systems, see [3], [9] and [10].

In this paper, a concept for an open-software template approach is proposed and evaluated based on its capability to detect the most relevant evasion scenarios. One of them is the diversion of fissile material. While passive gamma spectrometry is generally not well suited for determining the fissile mass in a nuclear component due to self-shielding effects, we examine conditions under which the technique is able to detect significant differences in the mass, in addition to isotopic composition and the size of the item's surface, in simple configurations using simulated gamma spectra.

\section{Validation}

Below, we will use extensive MCNP6 Monte Carlo simulations [11] to determine the expected performance of the inspection system. In order to validate the models used for these simulations, we draw on the high-quality SINBAD2013.12 benchmark data for the so-called beryllium-reflected plutonium (BeRP) ball. The dataset is part of a larger collection available from the Radiation Safety Information Computational Center (RSICC) [12]. The BeRP ball is a solid 4484-gram sphere of alpha-phase, weapon-grade plutonium (0.02 wt\% Pu238, 93.27 wt\% $\mathrm{Pu}-239,5.91$ wt\% $\mathrm{Pu}-240,0.07$ wt\% $\mathrm{Pu}-241,0.03$ wt\% $\mathrm{Pu}-242)$ 
enclosed in a thin steel casing. The ball was fabricated in 1980 and contained about $0.25 \mathrm{wt} \%$ of americium-241 at the time of the measurement (January 2009). The SINBAD exercise used a High-Purity Germanium (HPGe) detector for all gamma measurements $[13,14]$.

The simulated spectra are obtained using MCNP's pulse height tally. The following simulation procedure results in an accurate representation of the measured spectrum: First, a neutron simulation is run with a spontaneous fission source to determine induced fission rates as a function of the distance from the center of the BeRP ball. In a second simulation, induced fission reactions are not simulated during the neutron transport, but induced fission neutrons are directly included in the neutron source alongside spontaneous fission neutrons and the gamma rays from decay. The second step simulates only one source particle per simulation history. The partition into two steps is necessary, because simulating a spontaneous fission event and all subsequent interactions in one history could result in more than one gamma ray being detected per history. The pulse height tally yields the energy deposited per history, i.e., not per gamma ray [11, p. 3-177]. Tally data are broadened following the MCNP simulation using a normal distribution with a width (resolution) determined by the simulated detector type.

In order to increase the efficiency of the Monte Carlo simulations, we place a number of identical detectors on a spherical surface. Detectors can be evenly distributed on this surface using a modification of "Vogel's Method" [15]. Particles that reach one of the detectors but are not absorbed are terminated by 
the simulation to avoid the possibility of a non-realistic interaction in another detector. In order to further increase the simulation efficiency, we only include gamma energies above $100 \mathrm{keV}$ because lower-energy gammas would be easily shielded and do not provide a robust signature for verification purposes. A typical run corresponding to a 60-minute measurement uses about 1000 hours of single-CPU computer time.

Figure 1 shows a comparison between the experimental data acquired during the SINBAD measurement campaign and the results obtained with MCNP computer simulations. In the measurement shown, the BeRP ball was surrounded by a series of nesting spherical shells of high-density polyethylene, overall $7.62 \mathrm{~cm}$ thick. Neutrons are moderated and subsequently captured by hydrogen in these shells, resulting in the emission of high-energy gammas $(2223 \mathrm{keV})$ - an effect that is further discussed below.

\section{Concept}

In order to limit the amount of sensitive information acquired during a measurement, we examine whether robust results can be obtained with lowresolution detectors. While the information barrier concept is introduced to prevent inspectors from accessing the spectral data, acquiring low-resolution spectra can add an additional layer of security. ${ }^{1}$ Similar to Sandia's TRIS, the

\footnotetext{
${ }^{1}$ Even with low-resolution data, a knowledgeable inspector (for example, from another weapon state) could attempt a "dictionary attack," in which possible configurations based on "educated guesses" are forward-modeled until a matching spectrum is found.
} 


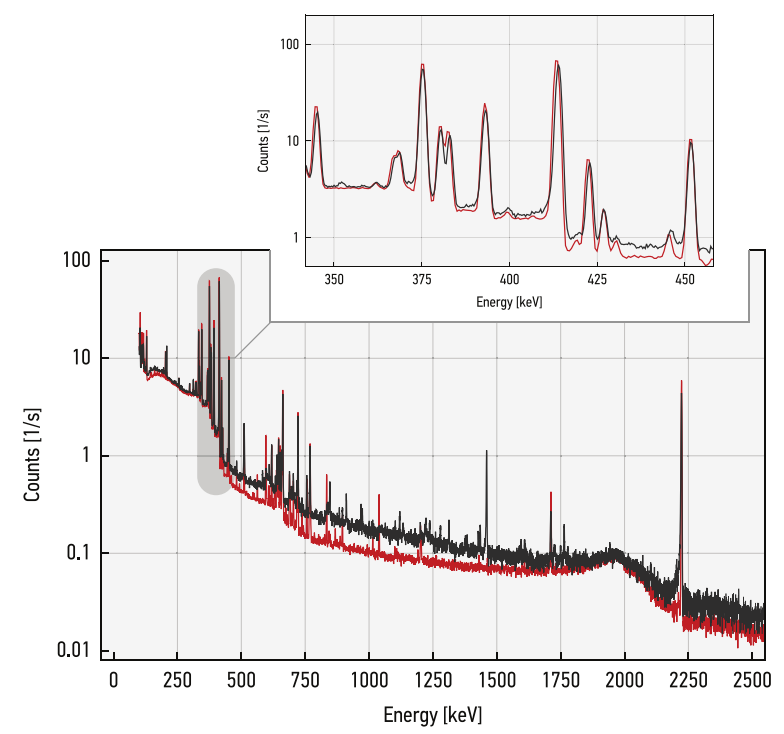

Figure 1: Measured (black) and simulated (red) high-resolution benchmark gamma spectra for the BeRP ball in a 7.62-cm polyethylene reflector. While the simulated background is overall slightly lower, the spectral features are well reproduced. Simulated (tally) data are broadened following the MCNP simulation to reproduce the resolution of the detector type used in the experiment (see insert of the 350-450 keV region). Note the peak at $2223 \mathrm{keV}$, which is due to neutron capture in polyethylene.

proposed setup uses a sodium-iodide (NaI) detector with a $5.08-\mathrm{cm}$ by $5.08-\mathrm{cm}$ crystal (Figure 2). The detector resolution has been determined by measurements of various radioactive sources with the Canberra Model $802 \mathrm{NaI}$ detector [16], and the respective parameters are used for subsequent MCNP calculations to broaden gamma lines. A 15-cm-thick polyethylene mask is placed between the source and the detector; this mask enables sensitivity to the neutron signature of the item via gamma detection from neutron capture in hydrogen. As the polyethylene also acts as gamma shielding, the mask has a central hole to allow 
gammas to pass through without attenuation. A 5-cm-thick steel plate (with a corresponding hole) serves to attenuate gammas that would otherwise scatter in the polyethylene and arrive in the detector, inter alia contributing to Compton background. The mask has an outer radius of $20 \mathrm{~cm}$ and a polyethylene mass of $17.6 \mathrm{~kg}$. The detector is positioned $10 \mathrm{~cm}$ behind the plate. The mask's thickness and its distance to the detector are chosen based on MCNP simulations performed to identify a geometry to yield a sufficient $2223-\mathrm{keV}$ peak intensity.

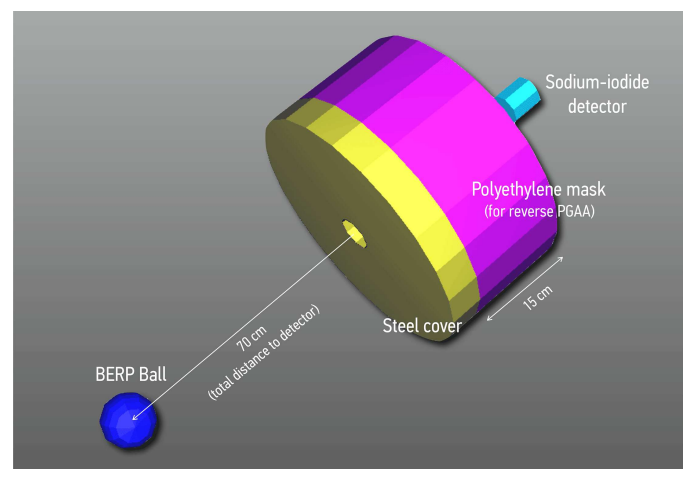

Figure 2: Simulated experimental setup for the multi-criteria template-matching approach.

(1)

Two principles guide the development of the system proposed here. First, we seek to avoid making assumptions about the expected radiation signatures; in particular, we are not trying to tailor the algorithms to particular spectral features of expected elements and nuclides such as uranium or plutonium. Candidate algorithms should be simple, versatile, and robust. Such an approach may also be easier to use and optimize in an unclassified setting because it should perform equally well with standard calibration sources. Second, we extend the standard template approach, which relies on a comparison of gamma 
spectra, by including two additional criteria in order to be more sensitive to important diversion scenarios that may otherwise be missed. Combined, the current criteria are:

Spectral features. The level of similarity between the acquired gamma spectrum and the template is determined by an "empirical" Kolmogorov-Smirnov (KS) test. In statistics, the standard two-sample KS test is a nonparametric hypothesis test that measures the similarity of two distributions. To do this, the test uses the largest absolute difference $\left(D_{\mathrm{KS}}\right)$ between the two cumulative distribution functions of the datasets as a measure of disagreement (Figure 3). It accepts the hypothesis that the distributions are equal if $D_{\mathrm{KS}}$ is less than a threshold value $D_{\mathrm{T}}$, which is determined by choosing a significance level $[17,18]$. The method has been previously and successfully applied to compare gamma spectra [19]. The function determining $D_{\mathrm{T}}$ for a given significance level assumes that both cumulative distribution functions are samples of the exact same parent distribution. In gamma spectrometry measurements, however, the measurement configuration, the detector voltage, and other experimental conditions inevitably vary to a small extent; the cumulative distribution functions of the reference item (template) and an identical inspected item are not samples of the exact same parent distribution. ${ }^{2}$ The hypothesis that two measured spectra

\footnotetext{
${ }^{2}$ In practice, we envision that the system is allowed to thermally stabilize before it is calibrated or before other measurements are made. Ideally, the template is recorded using a reference item immediately after an energy calibration has been performed. For optimum performance, the system can be recalibrated prior to each inspection to minimize the impact
} 
originate from the same source should, however, be accepted for insignificant variations. We therefore use an empirical KS test, which uses a large number of repeated measurements on a valid item to obtain the distribution of $D_{\mathrm{KS}}$ and guarantee a specified false-positive rate (for example, fewer than $1 \%$ of valid items are incorrectly flagged as invalid in a single measurement). ${ }^{3}$

Total gamma count rate. The features of a gamma spectrum are largely determined by the isotopics of the source and only weakly depend on the size of the object. In order to be directly sensitive to size, we therefore also compare the total gamma count rates observed for the template and the inspected item. To first order, these count rates scale with the projected surface area of the items (as seen by the detector) and provide a useful indicator for the similarity of their shapes.

Total neutron count rate. The gamma signature of massive objects, i.e., objects with thicknesses that exceed several millimeters, is dominated by the isotopics near the surface. The signature is much less sensitive to the presence of radioactive material away from the surface. For this reason, simple gamma measurements will not be robust for estimating the mass of an inspected item; at best, they can provide a minimum-mass estimate [3, p. 38-39]. Most diversion scenarios involve removing fissile material from an item, and an indicator that scales with mass would therefore be highly valuable. Here, we use neutron

of detector drift. We have experimentally confirmed that the proposed procedure is robust even for extended measurement times.

${ }^{3}$ See $[20]$ for a discussion of optimum repetition strategies for inspections. 

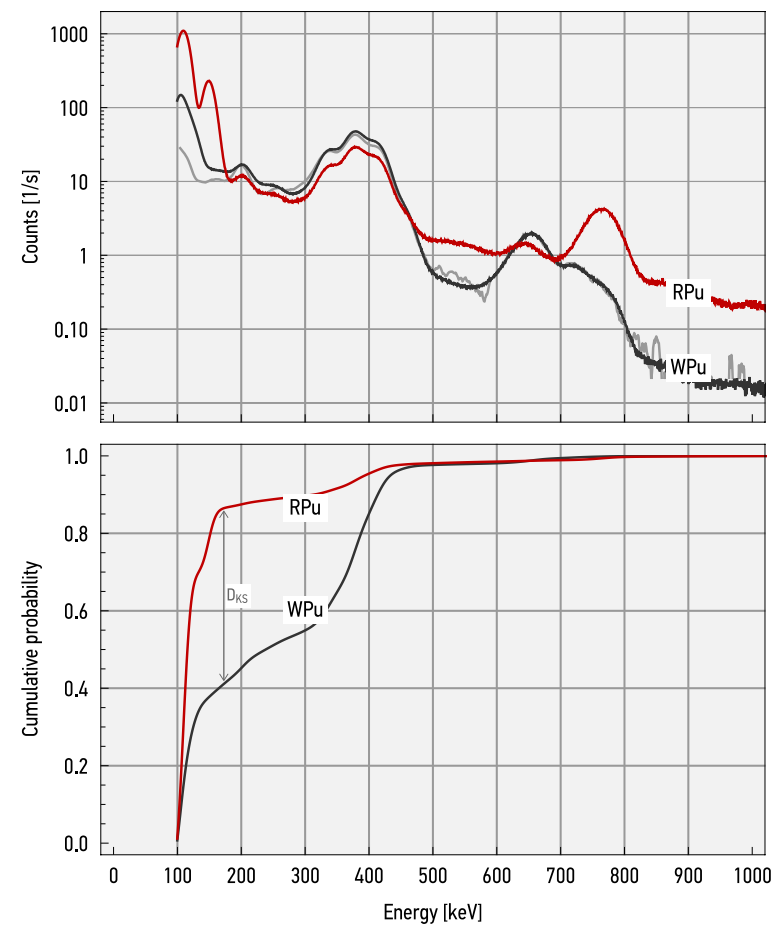

Figure 3: Quantifying the differences between two radiation spectra. Simulated gamma spectrum of the original BeRP ball compared against the spectrum of a similar ball made of reactor-grade plutonium (top, shown in black and red). The cumulative distribution function is equivalent to the fraction of the counts observed as a function of energy, and the maximum distance found in this distribution can be used to characterize the similarity of the spectra (bottom). For reference purposes, results from a measurement on the BeRP ball are also shown, though the experimental setup was slightly different from the modeled one (top, shown in gray).

\footnotetext{
${ }^{4}$ This works particularly well for plutonium components, but could be adapted for uranium components when combined with a small "driver source." Also, in a real configuration, neu-
} 
system as simple as possible, one measurement using a single type of detector is preferable. To do this, we use Prompt Gamma Neutron Activation Analysis (PGNAA) [21] in reverse and place the polyethylene mask shown in Figure 2 between the source and the detector. This mask thermalizes neutrons emanating from the inspected item and converts them into high-energy gammas $(2223 \mathrm{keV})$ following neutron capture in hydrogen [22]. The intensity of this gamma ray will be used as an indicator scaling with the mass of the object (Figure 4). If neutron multiplication in the inspected item is significant, then the dependency will be nonlinear. Since, for the template approach, we only compare the equivalence of radiation signatures and are not interested in absolute mass measurements, understanding the functional relationship is not necessary.

\section{Results}

To evaluate the viability of the proposed method, we examine a series of diversion scenarios from the (bare) BeRP ball. These include scenarios in which the isotopics and the apparent size of the object don't match the values of the reference item. More importantly, we also examine the sensitivity of the inspection system to material diversions while the outer dimensions of the component remain unchanged (Figure 5). To achieve this, in the simulations, material is gradually removed from the interior of the BeRP ball, which is difficult to detect with standard gamma spectrometry.

Table 1 summarizes the results of the empirical KS test for a series of com-

trons from a plutonium component could drive fission events in a nearby uranium component. 


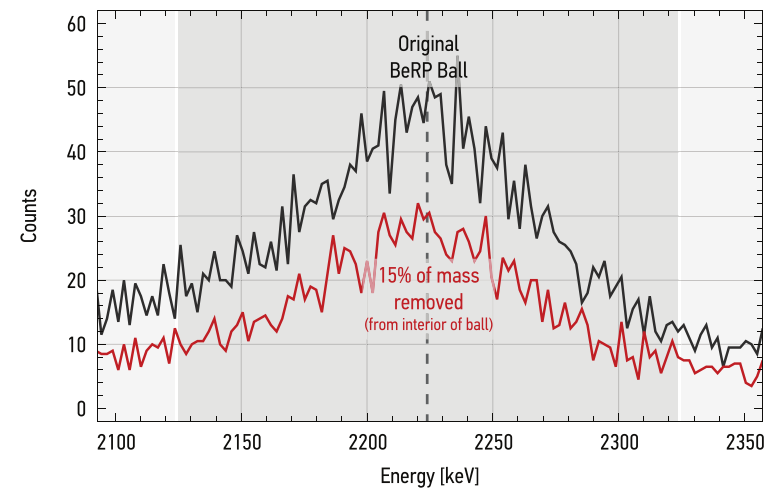

Figure 4: Gamma peak at $2223 \mathrm{keV}$ following neutron absorption in hydrogen. Shown are the simulated detector counts for the solid BeRP ball and for a hollow ball from which $15 \%$ of the material has been removed (3830 grams vs 4484 grams). The relative peak area drops by about $40 \%$ due to reduced mass and lower neutron multiplication in the hollow item. Shown absolute counts are for (simulated) 30-minute measurements.

parisons for configurations similar to those shown in Figure 5. In principle, many removals from the interior of a spherical shell are detectable in simulated measurements, but the respective $D_{\mathrm{KS}}$ values can be very small even when statistical noise is insignificant, i.e., for high count rates and/or long measurement times. In supplementary measurements using standard calibration sources, we have determined that, under real-world experimental conditions, a threshold

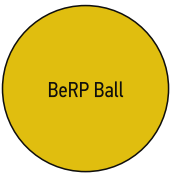

4484 grams (solid, $38 \mathrm{~mm}$ radius)

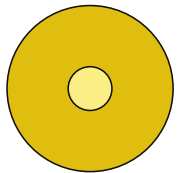

4400 grams (28 mm thickness)

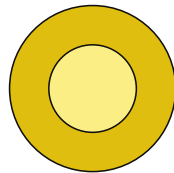

3830 grams (18 mm thickness)

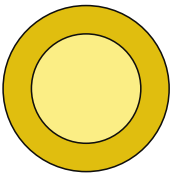

3200 grams (13 mm thickness)

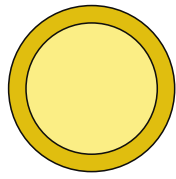

2270 grams (8 mm thickness)

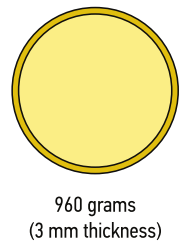

Figure 5: Diversion scenarios for the BeRP ball. 


\begin{tabular}{|c||c|c|c|c|c|c|}
\hline \multicolumn{1}{|c||}{} & \multicolumn{6}{c|}{ Reference item (Template) } \\
\hline & $4480 \mathrm{~g}$ & $4400 \mathrm{~g}$ & $3830 \mathrm{~g}$ & $3200 \mathrm{~g}$ & $2270 \mathrm{~g}$ & $960 \mathrm{~g}$ \\
\hline \hline $4480 \mathrm{~g}$ & (match) & - & - & - & - & - \\
\hline $4400 \mathrm{~g}$ & 0.0004 & (match) & - & - & - & - \\
\hline $3830 \mathrm{~g}$ & 0.0015 & 0.0012 & (match) & - & - & - \\
\hline $3200 \mathrm{~g}$ & 0.0021 & 0.0018 & 0.0006 & $($ match) & - & - \\
\hline $2270 \mathrm{~g}$ & 0.0029 & 0.0026 & 0.0015 & 0.0009 & $($ match) & - \\
\hline $960 \mathrm{~g}$ & 0.0069 & 0.0066 & 0.0055 & 0.0050 & 0.0044 & (match) \\
\hline
\end{tabular}

Table 1: Simulated $D_{\mathrm{KS}}$ values for comparisons between spherical shells with identical outer dimensions but different masses, i.e., with material removed from the interior (see Figure 5 for geometries). As expected, $D_{\mathrm{KS}}$ values increase with the amount of material removed, but small differences $\left(D_{\mathrm{KS}}<0.0010\right)$ are likely to be masked by experimental conditions and statistical noise for shorter measurement times (see text).

Despite its limited usefulness of detecting diversions from the interior of 232 an item that has the same outer geometry as a reference item, spectral features 
remain a powerful indicator for the comparison of the radiation spectra acquired for an inspected item and the template. Figure 6 shows the results of the empirical KS-test for a number of simulated five-minute measurements when the isotopics of the items differ. In this example, the $\mathrm{Pu}-240$ content in the inspected item is increased by $5 \%$ compared to the template (with a respective decrease in the $\mathrm{Pu}-239$ content). Even for this rather small isotopic change, the KS test returns significantly higher $D_{\mathrm{KS}}$ values with a mean of about 0.017 ; these values are easily distinguished from the valid case. ${ }^{5}$

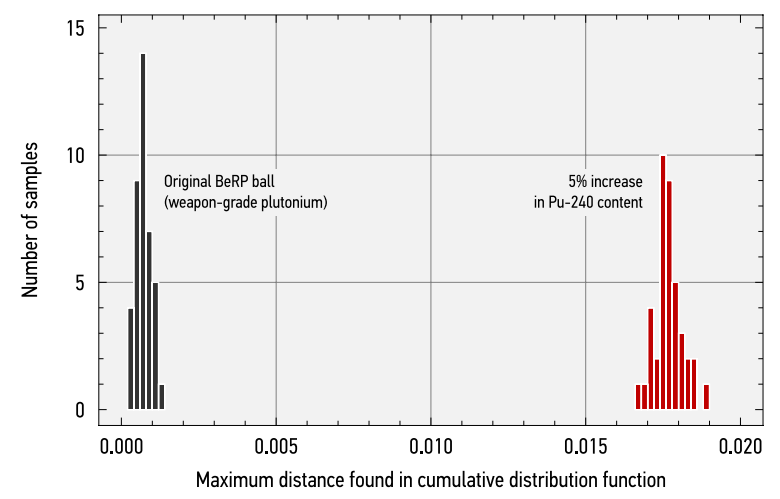

Figure 6: Empirical KS test for spectral features. Shown are results for 50 simulated fiveminute measurements with about 3,000,000 detector events each.

Total gamma counts are used as the second criterion, and Figure 7 summarizes the main results. The total counts are largely determined by two factors,

\footnotetext{
${ }^{5}$ For more drastic changes in the isotopics, as shown for example in Figure 3, the observed $D_{\mathrm{KS}}$ values will be significantly larger. In the case of weapon-grade versus reactor-grade plutonium, $D_{\mathrm{KS}} \approx 0.450$.
} 
the projected surface area (seen by the detector) and the isotopic composition of the item near the surface. Figure 7 shows results for an item that has the same mass as the original BeRP ball but a larger outer diameter and results for a $5 \%$ change in $\mathrm{Pu}-240$ content. Even small differences in these features are easily detected. Accordingly, the same total counts could be obtained by simultaneously changing both surface and isotopics. Changed isotopics would, however, be detected by the empirical KS test. In contrast, total gamma counts are not particularly sensitive to the interior of the item. As shown in Figure 7, the total count decreases marginally even for scenarios where on the order of $50 \%$ of the BeRP ball's mass have been removed. Only when the thickness of the shell drops below $5-7$ millimeters, the decrease becomes more significant. To be sensitive to the scenarios shown in Figure 5 and Table 1, an extra indicator is needed.

Neutrons emitted from the item are converted to high-energy (2223 keV) gammas via neutron capture in hydrogen. As illustrated in Figure 4, we use a 200-keV region-of-interest centered around this peak and use the total number of counts in that region as our third indicator. Figure 8 summarizes the results. As expected, items that have the same mass but different sizes may be difficult to distinguish based on their neutron signature. Figure 8 shows that this indicator is sensitive both to mass differences between two plutonium components and changes in the $\mathrm{Pu}-240$ isotopics, as spontaneous fission neutrons, which induce further fission events in the item, primarily come from $\mathrm{Pu}-240$. Accordingly, the same peak counts could be obtained by simultaneously changing both fissile 


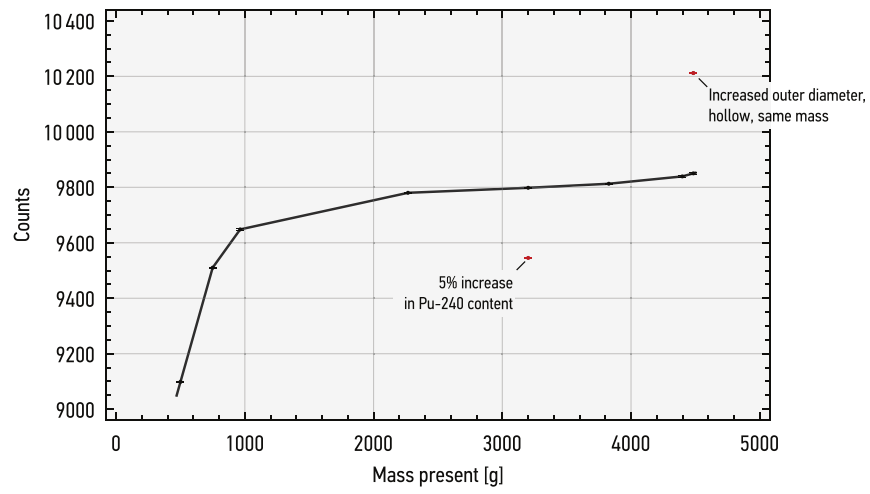

Figure 7: Total gamma counts per second as function of the mass present. Using the total count rate, it is difficult to distinguish the solid BeRP ball from a shell as long as the thickness of the shell exceeds $10 \mathrm{~mm}$ (40\% diversion with a remaining mass of about 2700 grams). The total count rate is most sensitive to projected surface area (geometry changes) and isotopics. Shown error bars are for simulated 30-minute measurements.

mass and isotopics. As changed isotopics would, however, be detected by the KS test, the proposed method is able to reject items with material withdrawn from the interior. ${ }^{6}$

\section{Discussion and Outlook}

In this article, we propose a measurement approach to support treaty verification applications based on low-resolution gamma-spectrometry for use in a

\footnotetext{
${ }^{6}$ An adversary could colocate a concealed neutron source with the inspected item in an attempt to reproduce the total neutron count rate observed for the reference item; this strategy would, however, run the risk of generating additional detectable signatures; for example, an extra neutron source could affect features of the gamma spectrum.
} 


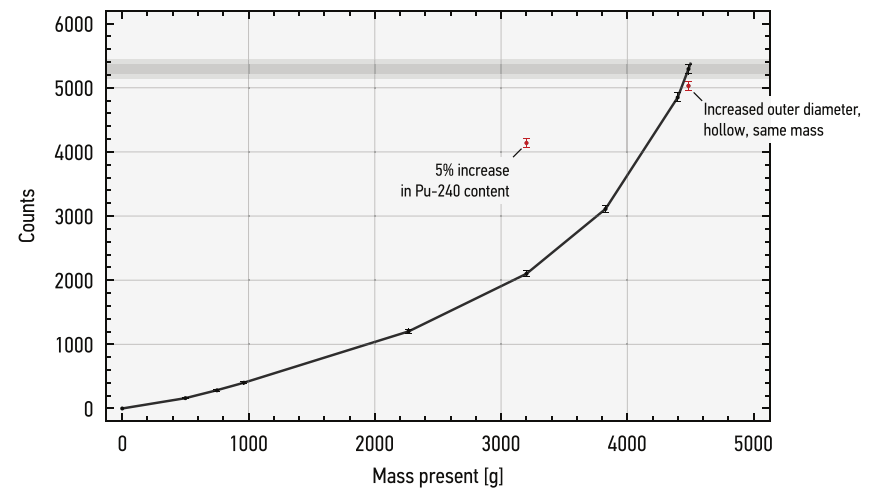

Figure 8: Total neutron counts as a function of the mass present. The nonlinearity is due to the increasing neutron multiplication as the mass of the item increases, i.e., as the size of the cavity in the item decreases. 4484 grams corresponds to the solid BeRB ball. Also shown are two additional diversion scenarios. Shown absolute counts are for simulated 30-minute measurements.

template-matching information barrier. The goal is to increase the robustness against evasion attempts compared to previously built systems. In particular, we propose the use of multi-criteria templates to simultaneously detect changes in isotopics, mass, and size or shape. As summarized in Table 2, each indicator can be optimized for one particular attribute, but it will often also be sensitive to other relevant characteristics of the inspected item, making cheating particularly difficult. As one specific implementation of the proposed concept, our analysis shows that the combination of an empirical Kolmogorov Smirnov test, the total gamma count rate test, and the total neutron count rate test (combined with a polyethylene mask) constitute an effective concept to distinguish valid from invalid plutonium items. 


\begin{tabular}{|c|c|c|c|}
\hline & MODIFIED KS TEST & TOTAL GAMMA & TOTAL NEUTRONS \\
\hline ISOTOPICS & Excellent indicator & $\begin{array}{c}\text { Good indicator } \\
\text { (in combination with others) }\end{array}$ & $\begin{array}{c}\text { Good indicator } \\
\text { (in combination with others) }\end{array}$ \\
\hline $\begin{array}{c}\text { SIZE } \\
\text { (PROJECTED SURFACE) }\end{array}$ & $\begin{array}{c}\text { Good indicator } \\
\text { (in combination with others) }\end{array}$ & $\begin{array}{c}\text { Excellent indicator } \\
\text { (in combination with others) }\end{array}$ & Inadequate \\
\hline MASS & Weak indicator & Weak indicator & $\begin{array}{c}\text { Excellent indicator } \\
\text { (in combination with others) }\end{array}$ \\
\hline
\end{tabular}

Table 2: Summary of the capabilities of our proposed system to detect differences in isotopic composition, projected surface area (size), and plutonium mass.

As a next step, extensive measurement campaigns (ideally with special nuclear materials, with both high and low neutron emission rates) will help provide a more nuanced understanding of systematic uncertainties from factors that typically cannot be taken into account in the simulations, as for example noise from the experimental equipment or small variations in the placement of the inspected item and the detector.

Our information barrier concept intentionally avoids the necessity of assumptions about specific spectral features, such as looking for gamma peaks of specific isotopes. This approach is followed in order to facilitate a fully unclassified discussion, which we believe is necessary for the successful implementation of a multinational verification regime. Furthermore, openness and transparency are essential principles to optimize the robustness of information barriers, as it enables cooperative research. Interested participants will then have the possibility to peer review and improve the algorithms ${ }^{7}$ or use their own algorithms

\footnotetext{
${ }^{7}$ The $\mathrm{C}++$ and Python code used for the first prototype of the device (Information Barrier
} 
for benchmarking purposes, contributing to an inclusive scientific discourse on this matter.

Acknowledgement. In the course of this project, we used more than two decades of single-CPU computer time. The authors thank the team of Princeton University's High Performance Cluster for their support. We also thank John Mattingly for valuable feedback on our modeling approach. This work is supported by the Consortium for Verification Technology (CVT) under Department of Energy National Nuclear Security Administration Award DE-NA 0002534. Experimental results shown in Figure 3 were obtained in measurements organized by the CVT at the Device Assembly Facility at the Nevada National Security Site in July 2016.

\section{References}

[1] Nuclear Posture Review Report, United States Department of Defense, Washington, DC, April 2010.

[2] Christine Comley, Mike Comley, Peter Eggins, Garry George, Steve Holloway, Martin Ley, Paul Thompson and Keith Warburton, Confidence, Security $\&$ Verification: The Challenge of Global Nuclear Weapons Arms Control, Atomic Weapons Establishment, AWE/TR/2000/001, Aldermaston, United Kingdom, 2000.

eXperimental, IBX) used to generate some of the results presented in this article is available at github.com/nuclearfutureslab/ibx. 
[3] David Spears (ed.), Technology RËD for Arms Control, U.S. Department of Energy, Office of Nonproliferation Research and Engineering, Washington, DC, 2001.

[4] Joint U.S.-U.K. Report on Technical Cooperation for Arms Control, United States Department of Energy, National Nuclear Security Administration, Washington, DC, May 2015.

[5] Dean J. Mitchell and Keith M. Tolk, Trusted Radiation Attribute Demonstration System, Sandia National Laboatories, Albuquerque, New Mexico, 2000.

[6] Sergey Razinkov, Alexander Livke, Sergey Kondratov, Duncan MacArthur, and Jonathan Thron, The Design and Implementation of the AVNG, Los Alamos National Laboratory, LA-UR-10-02623, Los Alamos, New Mexico, 2010.

[7] The United Kingdom-Norway Initiative: Further Research into the Verification of Nuclear Warhead Dismantlement, NPT/CONF.2015/WP.31, 2015 Review Conference of the Parties to the Treaty on the NonProliferation of Nuclear Weapons, United Nations, New York, 22 April 2015.

[8] Keith M. Tolk et al., "Trusted Radiation Inspection System," 42nd Annual INMM Meeting, Indian Wells, CA, July 2001.

[9] Malte Göttsche and Gerald Kirchner, "Measurement Techniques for War- 
head Authentication with Attributes: Advantages and Limitations," Science $\&$ Global Security, Vol. 22, 2014, pp. 83-110.

[10] Yan Jie and Alexander Glaser, "Nuclear Warhead Verification: A Review of Attribute and Template Systems," Science \& Global Security, Vol. 23, 2015, pp. 157-170.

[11] Denise Pelowitz (ed.), MCNP6 User's Manual, LA-CP-13-00634, Los Alamos National Laboratory, Los Alamos, New Mexico, May 2013.

[12] Shielding Integral Benchmark Archive and Database, Version December 2013, SINBAD-2013.12.

[13] John Mattingly, Polyethylene-Reflected Plutonium Metal Sphere: Subcritical Neutron and Gamma Measurements, SAND2009-5804, Revision 1, Sandia National Laboratory, Albuquerque, New Mexico, November 2009.

[14] John Mattingly and Dean J. Mitchell, "Implementation and Testing of a Multivariate Inverse Radiation Transport Solver," Applied Radiation and Isotopes, 70, 2012, pp. 1136-1140.

[15] Helmut Vogel, "A Better Way to Construct the Sunflower Head," Mathematical Biosciences, 44 (3-4), 1979, pp. 179-189.

[16] Canberra, Model 802 Scintillation Detectors Datasheet, CSP0232, 2009.

[17] W. Feller, "On the Kolmogorov-Smirnov Limit Theorems for Empirical Distributions," Ann. Math. Statist., 19 (2), 1948, 177-189. 
[18] Bernard W. Lindgren and Gayle W. McElrath, Introduction to Probability and Statistics, MacMillan, London, 1959.

[19] Alex Reinhart et al., "Detecting Changes in Maps of Gamma Spectra with Kolmogorov-Smirnov Tests," Nuclear Instruments and Methods A, 802, 2015, pp. 31-37.

[20] M. Kütt, S. Philippe, B. Barak, A. Glaser, and R. J. Goldston, "Authenticating Nuclear Warheads With High Confidence," 55th Annual INMM Meeting, Atlanta, Georgia, July 2014.

[21] Tamas Belgya, "Prompt Gamma Activation Analysis at the Budapest Research Reactor," Physics Procedia, 31, 2012, pp. 99-109.

[22] H. D. Choi et al., Database of Prompt Gamma Rays from Slow Neutron Capture for Elemental Analysis, STI/PUB/1263, International Atomic Energy Agency, Vienna, 2007. 